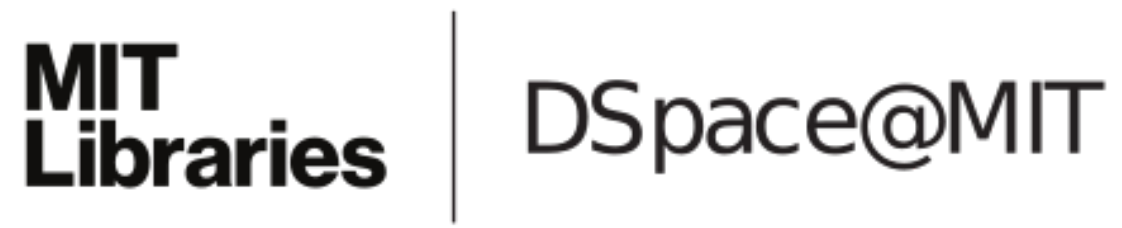

\author{
MIT Open Access Articles
}

Self-Aligned Acoustofluidic Particle Focusing and Patterning in Microfluidic Channels from Channel-Based Acoustic Waveguides

The MIT Faculty has made this article openly available. Please share how this access benefits you. Your story matters.

Citation: Collins, David J. et al. "Self-Aligned Acoustofluidic Particle Focusing and Patterning in Microfluidic Channels from Channel-Based Acoustic Waveguides." Physical Review Letters 120 , 7 (February 2018): 074502 @ 2018 American Physical Society

As Published: http://dx.doi.org/10.1103/PhysRevLett.120.074502

Publisher: American Physical Society

Persistent URL: http://hdl.handle.net/1721.1/114386

Version: Final published version: final published article, as it appeared in a journal, conference proceedings, or other formally published context

Terms of Use: Article is made available in accordance with the publisher's policy and may be subject to US copyright law. Please refer to the publisher's site for terms of use. 


\title{
Self-Aligned Acoustofluidic Particle Focusing and Patterning in Microfluidic Channels from Channel-Based Acoustic Waveguides
}

\author{
David J. Collins, ${ }^{1,3,4}$ Richard O’Rorke, ${ }^{1}$ Citsabehsan Devendran, ${ }^{2}$ Zhichao Ma, ${ }^{1}$ Jongyoon Han,,${ }^{3,4,5}$ \\ Adrian Neild, ${ }^{2}$ and $\mathrm{Ye} \mathrm{Ai}^{1, *}$ \\ ${ }^{1}$ Pillar of Engineering Product Development, Singapore University of Technology and Design, Singapore 487372, Singapore \\ ${ }^{2}$ Department of Mechanical and Aerospace Engineering, Monash University, Melbourne, Victoria 3800, Australia \\ ${ }^{3}$ Department of Biological Engineering, Massachusetts Institute of Technology, Cambridge, Massachusetts 02139, USA \\ ${ }^{4}$ Department of Electrical Engineering and Computer Science, Massachusetts Institute of Technology, \\ Cambridge, Massachusetts 02139, USA \\ ${ }^{5}$ BioSystems and Micromechanics (BioSyM) IRG, Singapore-MIT Alliance for Research and Technology (SMART) Centre, \\ Singapore 138102, Singapore
}

(Received 25 October 2017; published 15 February 2018)

\begin{abstract}
Acoustic fields have been widely used for manipulation of particles and cells within microfluidic systems. In this Letter, we explore a novel acoustofluidic phenomenon for particle patterning and focusing, where a periodic acoustic pressure field is produced parallel to internal channel boundaries with the imposition of either a traveling or standing surface acoustic wave (SAW). This effect results from the propagation and intersection of edge waves from the channel walls according to the Huygens-Fresnel principle and classical wave fronts from the substrate-fluid interface. We demonstrate versatile control over this effect to produce both one- and two-dimensional acoustic patterning from one-dimensional SAW fields and its utility for continuous particle focusing. Uniquely, this channel-guided acoustic focusing permits the generation of robust acoustic fields without channel resonance conditions and particle focusing positions that are difficult or impossible to produce otherwise.
\end{abstract}

DOI: 10.1103/PhysRevLett.120.074502

High frequency acoustic fields are a promising noncontact alternative for the manipulation of microscale objects. Applications include the patterning and sorting of cells and particles in industry and clinical settings, where strong yet biocompatible [1,2] acoustic forces result in rapid movement and/or stable trapping of cells and other bioparticles in both liquids [3] and gases [4] for continuous sorting [5-7] and cell manipulation [8-10]. To generate the desired particle behavior, the acoustic field must be appropriately shaped, e.g., with the required spatially localized phase and wavelength properties. While it is possible to shape the wave fronts emanating from a monolithic acoustic transducer by shaping the transducer itself $[11,12]$, refined acoustofluidic manipulation in specific microfluidic channel regions often requires the careful alignment and/or integration of the acoustic source with the microfluidic device. Surface acoustic waves (SAWs) are one such actuation technology that permits localized acoustic fields from micropatterned transducers [13-17], including those with electrodes designed to create shaped acoustic beams [18-20]. Superimposing two orthogonal standing wave fields from four transducers, for example, generates a time-averaged two-dimensional field $[21,22]$ for movement [23], 3D manipulation [24], and single-cell patterning [1]. Acoustic metamaterials [25], holographic intermediaries [26], phase variable fields [27], and phononic structures [28-30] are suitable avenues for shaping such fields. A typical SAW device, however, generates an acoustic field in a fluid mostly irrespective of the overlaying channel elements. As a result, manipulating particles into specific channel locations requires precise alignment to position acoustic force potential minima [31], independent phase tuning of opposing transducers [32], or channel resonance [33]. Waveguide features, typically taking the form of a microstructured pillar bridging a gap between the substrate and a fluid channel, can be used to localize the wave transmission into defined regions [34-37]. The transmitted wave, however, still reflects the underlying substrate conditions, where the trapping locations above the pillar are a function of the substrate alignment in the case of a standing SAW [38].

It would be ideal if the acoustic field could be directly guided by microfluidic channel features, as in the case of optical waveguides [39]. This characteristic is integral to bulk acoustic wave (BAW) acoustofluidic devices [40-42], though the applied frequency of an affixed BAW transducer must correspond to resonance conditions within a microfluidic channel for effective manipulation, though impedance-matched materials can be used to circumvent this limitation [41]. Devendran et al. recently exploited diffractive effects to create lines of particles using a traveling wave with actuation by a single traveling SAW [43]. However, this 
work only examined the generation of acoustic fields where the channel was oriented explicitly parallel to the wave fronts and required very high aspect ratio channels $(\sim 100 \times)$ to isolate this effect. In this Letter, we demonstrate robust particle patterning from channel-based waveguides with conventional microchannel dimensions and unique manipulation that arises when opposing channel walls are oriented parallel to the wave front propagation direction. This effect results from the interference of spherical waves emanating from a channel-limited transducer region according to the Huygens-Fresnel principle. Using this principle, we generate time-averaged pressure fields that are effectively selfaligned with the channel features. Importantly, by decoupling the orientation of the acoustic field that is generated in the fluid from that on the substrate, a wide range of particle migration behaviors and complex acoustic fields become viable with the imposition of only one-dimensional SAWs. This notably includes the preferential migration of solid, dense particles to channel boundaries. Here we utilize this boundary-dependent acoustofluidic phenomenon for both two-dimensional particle pattern generation from a onedimensional standing wave and continuous particle focusing with a single traveling wave.

Both traveling and standing SAWs (TSAW and SSAW) can readily be used for microscale manipulation, where these fields result in migration away from the SAW transducer $[3,34,35,44]$ and toward minimum displacement locations on the substrate, [45] respectively. The common element with SAW types, however, is that particle migration is oriented along the axis of SAW propagation. In contrast, we demonstrate that the channel walls can generate periodic patterning perpendicular to the substrate wave fronts. In the Supplemental Material [46], Fig. S1 shows that the interference pattern above a finite transducer can be approximated by the intersection of cylindrical waves [47] from the channel edge and classical wave fronts emanating from the substrate into the fluid. In the case of a SAW, a finite transducer extent can be created with the imposition of channel boundaries, where the acoustic amplitude at a given point in a fluid is equal to the sum of all contributing spherically propagating waves. The concept of total internal reflection prevents acoustic energy arising from certain regions on the substrate under the solid channel wall, where a SAW is also present, from influencing the field in the fluid domain [48]. Acoustic energy can either reflect at or refract across a boundary between two materials, with dissimilar sound speeds with different incoming and outgoing angles of incidence, where $\theta_{1}$ is the angle at which an acoustic wave intersects the interface. According to Snell's law, there exists a critical angle $\theta_{c}$ for the angle $\theta_{1}$ above which the incident energy is entirely reflected. This principle is illustrated in Fig. 1(a), which plots the time-averaged pressure field near a single channel wall when actuated by a TSAW, which attenuates along the substrate-polydimethylsiloxane (PDMS) and substrate-water interfaces. For PDMS and
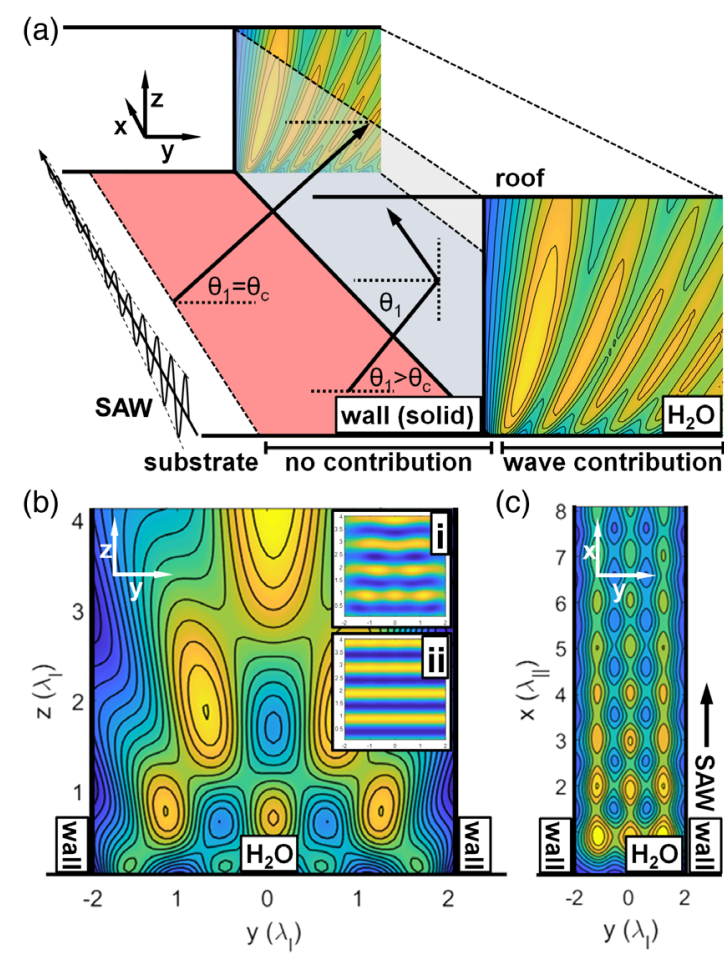

FIG. 1. Principle of channel-based acoustofluidic waveguides. (a) A time-averaged pressure distribution occurs near the boundaries of a spatially finite wave source in the $x-y$ plane. With a SAW, this is readily achieved by affixing a channel wall to the substrate. The acoustic pressure field near a single channel wall is shown. (b) Placing two channel walls near one another yields a complex acoustic field, here mapped for a wave source with a width of $4 \lambda_{l}$ in the $y-z$ plane. Insets show (i) a time-domain image of the pressure field with a $4 \lambda_{l}$ and (ii) $100 \lambda_{l}$ transducer width over the same domain. (c) In the $y$ - $x$ plane (at $h=\lambda_{l}$ ), periodicity develops parallel to the channel waveguides.

water $\left(c_{\mathrm{PDMS}}=1030 \mathrm{~m} / \mathrm{s}\right.$ and $c_{l}=1500 \mathrm{~m} / \mathrm{s}$, respectively $)$ this yields $\theta_{c}=43^{\circ}$. Bringing two channel walls in proximity to one another results in distinct two-dimensional nodal positions in the $y$-z plane; Fig. 1(b) shows this acoustic field for a fluid domain with a height and a transducer width of $4 \lambda_{l}$, where $\lambda_{l}$ is the acoustic wavelength in the fluid. The Fig. 1(b) inset examines the effects of limiting the transducer extent, where (i) shows the transient time-domain wave fronts emanating from a $4 \lambda_{l}$ wide transducer and a (ii) $100 \lambda_{l}$ transducer; in the latter case, the wave fronts are nearly uniform in this $4 \times 4 \lambda_{l}$ domain. Examining the field in the $y-x$ plane (here for a height of $\lambda_{l}$ ) in Fig. 1(c), however, demonstrates how the channel walls act as an acoustic waveguide in the plane of the substrate, where a periodic field develops parallel to the channel wall and perpendicular to the acoustic wave fronts. Notably, we also recover the periodic spacing parallel to the acoustic wave fronts $\lambda_{\|}$as predicted elsewhere [43].

To determine the periodic spacing that occurs perpendicular to these wave fronts $\lambda_{\perp}$, we note that the effect of a spatially limited transducer is the production of 

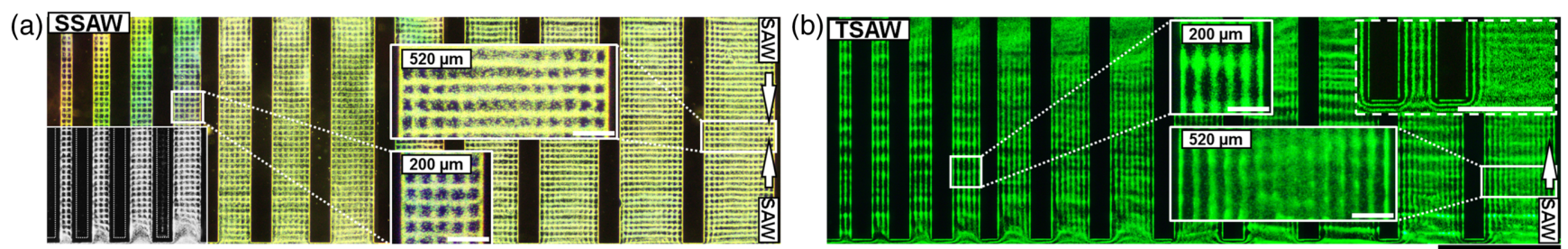

FIG. 2. Particle patterning via SAW and channel feature interactions. (a) Two-dimensional particle patterning is accomplished with a one-dimensional SSAW. Gray region in the bottom left incorporates air gaps in the channel walls, with no effect on nodal spacing vs solid PDMS walls. (b) Lateral patterning is independently observed with the imposition of a TSAW. Dashed inset (right) shows a channel feature at the edge of the domain, demonstrating patterning in an (effectively) unbounded domain (scale bar is $500 \mu \mathrm{m}$ ). Other insets show selected regions with 200 and $520 \mu \mathrm{m}$ widths. $\lambda_{\text {SAW }}=80 \mu \mathrm{m}$ and applied power $=1.6 \mathrm{~W}$. Scale bar (lower right) is $1 \mathrm{~mm}$. Left enlarged inset scale bars are $100 \mu \mathrm{m}$.

edge waves $[49,50]$. The interference of these with the classical wave fronts that propagate into the fluid at the Rayleigh (Ra) angle $\theta_{\mathrm{Ra}}$ from the SAW substrate [51] that yields an interference pattern in the $x-y$ plane above the transducer (Supplemental Material Fig. S2 [46]). In the case of a low channel height (smaller than a few acoustic wavelengths), the examination of these wave fronts near the substrate gives a good approximation of how these waves interact. The periodicity orthogonal to the SAW propagation direction $\lambda_{\perp}$ is given by the lateral spacing of these wave intersections. This spacing can readily be expressed in terms of trigonometric functions by

$$
\lambda_{\perp}=\lambda_{\mathrm{SAW}} \tan \left[\sin ^{-1}\left(\frac{\lambda_{l}}{\lambda_{\mathrm{SAW}}}\right)\right]
$$

for heights in the fluid $h \lesssim \lambda_{l}$. This can similarly be expressed in terms of the liquid and solid sound speeds (with the application of the appropriate trigonometric identities) by

$$
\lambda_{\perp}=\frac{\lambda_{l}}{\sqrt{1-\left(\frac{c_{l}}{c_{s}}\right)^{2}}} .
$$

The $\perp$ symbol denotes that the predicted spacing is for a channel that is perpendicular to the SAW wave fronts $\left(\theta=90^{\circ}\right)$. As the periodicity resulting from the channel wall is a function of $\theta$, defined as the orientation of the channel wall with respect to the wave propagation direction, we examine this $\theta=90^{\circ}$ case, as this is the only one that will result in equal spacings for parallel channel walls, therefore permitting channel waveguides and uniform particle pattern spacings. As discussed later, though patterning spacing is reflected by this simple model, it is necessary to simulate the system in three dimensions to show how the distance between opposing channel boundaries can influence $\lambda_{\perp}$.

Our system is comprised of a SAW actuator and a channel bonded to the underlying SAW substrate. The SAW device is composed of a piezoelectric $128^{\circ} y$-cut, $x$-propagating lithium niobate $\left(\mathrm{LN}, \mathrm{LiNbO}_{3}\right)$ substrate, with patterned interdigital transducer (IDT) electrode finger pairs on the surface. Devices with SAW wavelengths of $60,78,80$, and
$140 \mu \mathrm{m}(2,8,13.8$, and $8 \mathrm{~mm}$ apertures, respectively) and a slanted finger IDT device [20,52,53] with wavelengths between 40 and $80 \mu \mathrm{m}$ ( $7.8 \mathrm{~mm}$ aperture) were used in this study. For a detailed description on SAW devices for acoustofluidic applications, the reader is encouraged to read one of a few representative reviews on the topic [13-17,54,55]. A PDMS channel $22 \mu \mathrm{m}$ in height with $160 \mu \mathrm{m}$ wide channel features is bonded after oxygen plasma treatment of both surfaces (PDC 32G, Harrick Plasma, USA). The attenuation of the SAW prior to the fluidic channel is minimized with a $50 \mu \mathrm{m}$ wide channel wall.

Simulated pressure fields are obtained by discretizing the SAW-fluid interface into a grid of point sources for spherical waves and summing the spherical waves at each point in the liquid. In the $y-z$ plane, this simplifies to an integral of a spherical wave function across the acoustic aperture. In the $x-y$ plane, this becomes a convolution of the spherical wave function with an appropriate SAW displacement boundary condition. Here, we used the boundary conditions for SAW propagation beneath a liquid channel reported previously [56].

In these results, we demonstrate the use of channel features to produce particle pattering independent of the underlying acoustic wave nodal positions. Figure 2(a) shows the periodic spacing that develops with the imposition of both a SSAW and TSAW. As the standing wave is composed of two intersecting traveling wave components, each with identical $\lambda_{\perp}$, the corresponding $\lambda_{\perp}$ will be the same. Because of the dimensions of the channel shown ( $\approx 6.2 \mathrm{~mm}$ width), this image is composed of several stitched images obtained with a $4 \times$ objective. The gray region in the lower left of Fig. 2(a) is superimposed to show the effect of a hollow channel pillar feature (the dotted lines here represents the air-PDMS edge). Notably, a hollow pillar has negligible effect on the patterning, since the total internal reflection that occurs in the interior of a solid pillar is sufficient to produce the discontinuity in the substrate wave source. The spacing between neighboring channel features varies here between 80 and $520 \mu \mathrm{m}$, in increments of $40 \mu \mathrm{m}$, with decaying strength of the lateral patterning component for larger channel spacings. Figure 2(b) shows the same system with single-side IDT actuation, with the 
(a)

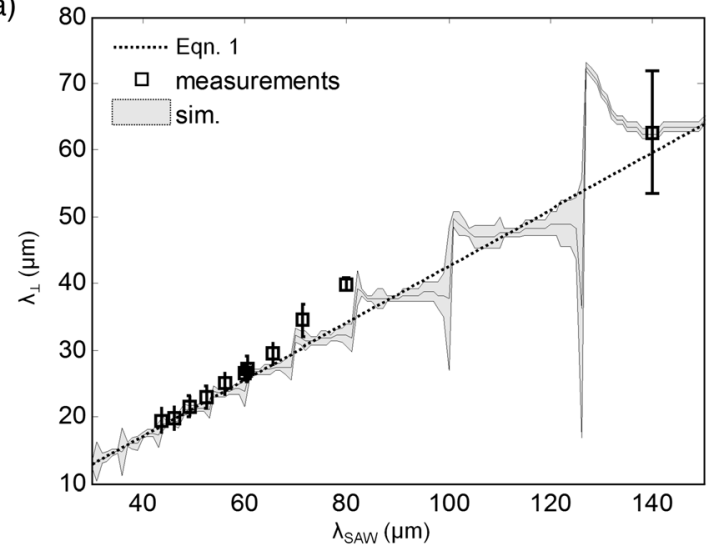

(b)

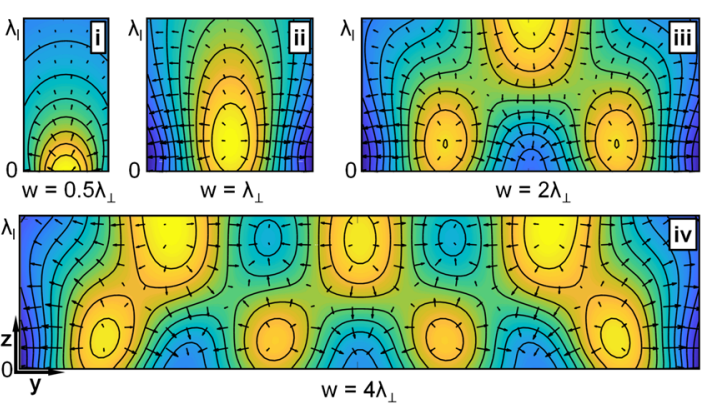

FIG. 3. Control over lateral periodicity. (a) The simulated periodicity perpendicular to the SAW propagation direction $\lambda_{\perp}$ matches the predictions from Eq. (1) and the experimental results. (b) Setting this width in terms of the calculated lateral periodicity, we show that the number of lateral node-antinode locations at a given height (here mapped from $z=0$ to $\lambda_{l}$ ) corresponds directly with the width when cast in terms of different $\lambda_{\perp}$.

TSAW propagating in the $+x$ direction. The left inset here shows the expected equivalent lateral spacing between SSAW and TSAW cases. The right inset shows that, while neighboring channel features are desirable for producing symmetrical particle patterning (occurring between the opposing channel elements), they are not a necessary condition for the generation of lateral periodicity. Here, the unbounded right edge (nearest channel edge is $1200 \mu \mathrm{m}$ to the right) of the channel feature nevertheless results in several (decaying) force potential minima, noted also in the $520 \mu \mathrm{m}$ wide pillar spacing (inset bottom). This is a consequence of the inverse relationship between wave front displacement amplitude and distance from the source (i.e., displacement magnitude in the liquid $\xi_{l} \propto r^{-1}$ ).

Figure 3(a) compares predicted, measured, and simulated periodicities in a $200 \mu \mathrm{m}$ wide channel across a range of acoustic wavelengths. The wavelengths reported here for the straight IDT devices $\left(\lambda_{\mathrm{SAW}}=60,80\right.$, and $\left.140 \mu \mathrm{m}\right)$ and the slanted IDT device $\left(\lambda_{\mathrm{SAW}} \approx 44,46,49,52,56,60,66\right.$, and $71 \mu \mathrm{m}$ ) correspond to the IDT pitches resulting in resonance at the given applied frequencies $(f=66,49$, and $28 \mathrm{MHz}$ for straight IDTs; $f=90,85,80,75,70,65,60$, and $55 \mathrm{MHz}$ for slanted IDT), as examined elsewhere [20]. Supplemental Material Fig. S3 [46] shows how the pattern
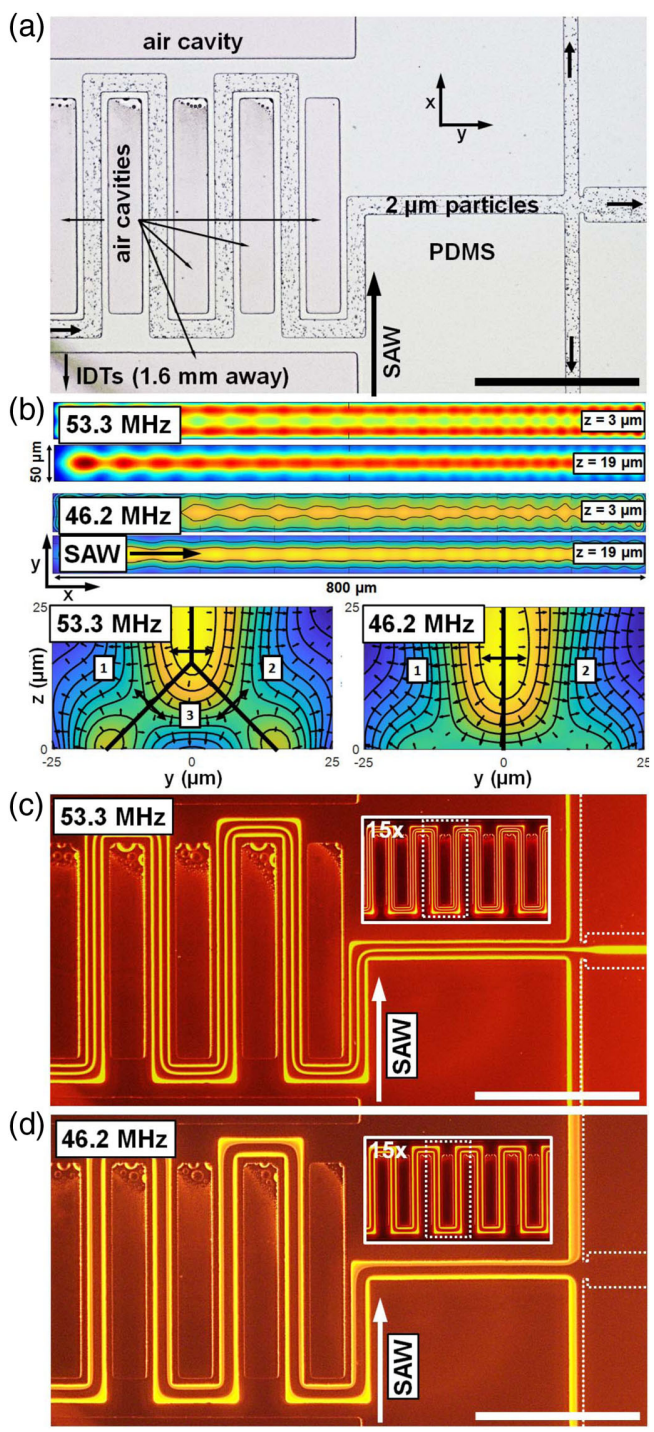

FIG. 4. Continuous particle focusing and negative sorting via channel-based waveguides. (a) A series of loops $(15 \times)$ with $800 \mu \mathrm{m}$ long focusing channels are subjected to SAWs to generate acoustic force gradients parallel to the channel waveguides. (b) The modeled acoustic force shows the appearance of either two or three local minima in the $x-y$ plane (upper view), assessed here at $z=3$ and $19 \mu \mathrm{m}$, and the $y-z$ plane (lower view) for two applied frequencies (53.3 and $46.2 \mathrm{MHz}$ ). (c) At $53.3 \mathrm{MHz}$, three lateral focusing positions develop [denoted as 1,2 , and 3 in (b)], with fluorescent $2 \mu \mathrm{m}$ particle migration to the center and edges of the channel. (d) At 46.2 MHz, all particles are pushed to the edges of the channel. Here shown for a $\lambda_{\mathrm{SAW}}=78 \mu \mathrm{m}, 100 \mathrm{~mW}$ applied power, and $2 \mu \mathrm{l} / \mathrm{min}$ flow rate. Scale bars are $500 \mu \mathrm{m}$.

periodicity in the $x$ and $y$ directions are examined independently for an example case with a $60 \mu \mathrm{m}$ SAW wavelength. In the $x$ direction, we extract the expected periodicity with particle aggregation in each nodal position along the substrate (every $\lambda_{\mathrm{SAW}} / 2$ ). While Eq. (1) provides a good first-order approximation of $\lambda_{\perp}$, the actual spacing in practice is subject to the precise ratio of the acoustic 
wavelength to the channel dimensions, which affects how the field evolves in the $y-z$ plane (examined later in Fig. 4). The simulated spacing in Fig. 3(a) assumes that particles aggregate in a nodal position one-half of a fluid wavelength from the channel roof, where a minimum time-averaged pressure condition develops in the $z$ direction. Figure 3(b) demonstrates the importance of the $\lambda_{\perp}$ parameter from Eq. (1) and our control over the acoustic field shape by taking this value into account, here plotted with a channel width of (i) $0.5 \lambda_{\perp}$, (ii) $\lambda_{\perp}$, (iii) $2 \lambda_{\perp}$, and (iv) $4 \lambda_{\perp}$. Arrows denote the direction of particle migration in this field. We see here the direct relationship between the width of the channel in perpendicular wavelengths and the number of pressure features in the $y$ direction at a given channel height; i.e., the number of pressure antinodes near the substrate is equal to the width in $\lambda_{\perp}$. This results in particle migration (i) away from the transducer, (ii) toward the channel walls, or (iii), (iv) more complex 2D patterning behavior, depending on the channel dimensions. Notably, cases (i) and (ii) are unique to this channel waveguide method as compared to most BAW and SAW standing wave results, as they set up either a rapidly decaying (in the $z$ direction) surface localized pressure field for a very narrow channel width $\left(w<\lambda_{\perp}\right)$ or nodal positions that are exclusively at the channel edges when the width is on the order of $\lambda_{\perp}$.

In (ii), the channel width is still smaller than the underlying SAW wavelength. As lateral particle positioning can be readily measured, we examine this case experimentally. In Fig. 4, we demonstrate continuous particle focusing using a SAW device with an IDT wavelength of $78 \mu \mathrm{m}$ above $(53.3 \mathrm{MHz})$ and below $(46.2 \mathrm{MHz})$ the design frequency of approximately $50 \mathrm{MHz}\left[f=c_{s} / \lambda_{\mathrm{SAW}}=(3931 \mathrm{~m} / \mathrm{s}) /(78 \mu \mathrm{m})\right]$ and within the calculated $3 \mathrm{~dB}$ bandwidth for this device $( \pm 4.4 \mathrm{MHz})$ [57]. Figure 4(a) shows an image of this device prior to excitation, where air cavities are used on either side of the channel features to minimize SAW attenuation. Figure 4(b) models the pressure distribution in an $800 \mu \mathrm{m}$ long channel section that is oriented parallel to the SAW propagation direction in the $x-y$ and $y-z$ planes, showing two local pressure minima in the case of a $46.2 \mathrm{MHz}$ signal $\left(\lambda_{\mathrm{SAW}} \approx 85 \mu \mathrm{m}\right)$ and three in the case of a $53.3 \mathrm{MHz}$ signal $\left(\lambda_{\mathrm{SAW}} \approx 74 \mu \mathrm{m}\right)$; this third minimum appears in the channel center and is visible in the $x-y$ plane in the $z=3 \mu \mathrm{m}$ image for this frequency. The experimental images in Figs. 4(c) and 4(d) are manifestations of these acoustic fields, where fluorescent $2 \mu \mathrm{m}$ polystyrene beads aggregate in the predicted local minima in a continuous flow, with a significant change in particle focusing behavior, despite a small change in applied frequency (see Video SM1 in the Supplemental Material [46]). As in other acoustofluidic systems, focusing performance at a given applied power is expected to degrade with increasing flow rate [58].

The results in Fig. 4 are unique for two important reasons. First, in contrast to half-wavelength resonators where nodal positions occur in the channel center [42,58,59], the inverse occurs here, with nodal positions adjoining channel boundaries. This permits the negative sorting of particles that are denser than the surrounding media, previously only possible with less dense specimens (i.e., fat cells) [60]. Second, this method promises to be more energetically efficient than comparable standing wave methods, which may only couple energy into the fluid over one half-wavelength [61]. Using a channel-based waveguide the length of the channel along the SAW propagation direction can be arbitrarily long; longer channel elements increase the proportion of substrate energy that couples into the overlaying fluid, where the translating effect (though not the intensity) of the acoustic field is relatively uniform along the channel length.

We have demonstrated the importance of channel boundaries for the generation of nontraditional pressure fields in acoustofluidic systems. By limiting the spatial extent of acoustic coupling with channel boundaries, we generate time-averaged acoustic fields whose alignment and positions are determined by channel locations. This permits acoustic fields that are self-aligned with channel features, bringing this important quality of conventional BAW transducers to SAW designs, while also allowing the use of materials compatible with soft lithography (as opposed to the etched silicon and glass devices used with BAWs). We utilize these fields for 1D and 2D patterning with the imposition of a 1D TSAW and SSAW, respectively, and further demonstrate its utility for continuous particle focusing. In this latter case, focusing occurs at the channel edges rather than in the center as with half-wavelength resonant channels $[58,62]$. This shows promise for expanding the range of acoustofluidic manipulations that can be performed, where hydrodynamic interactions with channel walls [63-65] are enhanced and total residence time is increased in the low fluid velocities near channel boundaries, offering additional time for chemical processing or optical detection.

We gratefully acknowledge the support received from Singapore Ministry of Education Academic Research Fund Tier 2 (T2MOE1603) awarded to Y. A. D. C. was partially supported by a SUTD-MIT Joint Postdoctoral Fellowship.

*Corresponding author. aiye@sutd.edu.sg

[1] D. J. Collins, B. Morahan, J. Garcia-Bustos, C. Doerig, M. Plebanski, and A. Neild, Nat. Commun. 6, 8686 (2015).

[2] M. Wiklund, Lab Chip 12, 2018 (2012).

[3] D. J. Collins, A. Neild, and Y. Ai, Lab Chip 16, 471 (2016).

[4] D. Foresti, M. Nabavi, M. Klingauf, A. Ferrari, and D. Poulikakos, Proc. Natl. Acad. Sci. U.S.A. 110, 12549 (2013).

[5] W. L. Ung, K. Mutafopulos, P. Spink, R. W. Rambach, T. Franke, and D. A. Weitz, Lab Chip 17, 4059 (2017).

[6] A. A. Nawaz, Y. Chen, N. Nama, R. H. Nissly, L. Ren, A. Ozcelik, L. Wang, J. P. McCoy, S. J. Levine, and T. J. Huang, Anal. Chem. 87, 12051 (2015). 
[7] Z. Ma, Y. Zhou, D. J. Collins, and Y. Ai, Lab Chip 17, 3176 (2017).

[8] Y. Kurashina, K. Takemura, and J. Friend, Lab Chip 17, 876 (2017).

[9] K. Chen, M. Wu, F. Guo, P. Li, C. Y. Chan, Z. Mao, S. Li, L. Ren, R. Zhang, and T. J. Huang, Lab Chip 16, 2636 (2016).

[10] D. J. Collins, B. L. Khoo, Z. Ma, A. Winkler, R. Weser, H. Schmidt, J. Han, and Y. Ai, Lab Chip 17, 1769 (2017).

[11] H.-S. Hsu, F. Zheng, Y. Li, C. Lee, Q. Zhou, and K. K. Shung, Appl. Phys. Lett. 101, 024105 (2012).

[12] J. Y. Hwang, J. Kim, J. M. Park, C. Lee, H. Jung, J. Lee, and K. K. Shung, Sci. Rep. 6, 27238 (2016).

[13] L. Y. Yeo and J. R. Friend, Annu. Rev. Fluid Mech. 46, 379 (2014).

[14] X. Ding, P. Li, S.-C. S. Lin, Z. S. Stratton, N. Nama, F. Guo, D. Slotcavage, X. Mao, J. Shi, and F. Costanzo, Lab Chip 13, 3626 (2013).

[15] T. Dung Luong and N. Trung Nguyen, Micro and Nanosyst. 2, 217 (2010).

[16] Z. Wang and J. Zhe, Lab Chip 11, 1280 (2011).

[17] G. Destgeer and H. J. Sung, Lab Chip 15, 2722 (2015).

[18] D. J. Collins, Z. Ma, J. Han, and Y. Ai, Lab Chip 17, 91 (2017).

[19] D. J. Collins, Z. Ma, and Y. Ai, Anal. Chem. 88, 5513 (2016).

[20] R. W. Rambach, J. Taiber, C. M. Scheck, C. Meyer, J. Reboud, J. M. Cooper, and T. Franke, Sci. Rep. 6, 21980 (2016).

[21] I. Bernard, A. A. Doinikov, P. Marmottant, D. Rabaud, C. Poulain, and P. Thibault, Lab Chip 17, 2470 (2017).

[22] L. Tian, N. Martin, P. G. Bassindale, A. J. Patil, M. Li, A. Barnes, B. W. Drinkwater, and S. Mann, Nat. Commun. 7, 13068 (2016).

[23] X. Ding, S.-C. S. Lin, B. Kiraly, H. Yue, S. Li, I.-K. Chiang, J. Shi, S. J. Benkovic, and T. J. Huang, Proc. Natl. Acad. Sci. U.S.A. 109, 11105 (2012).

[24] D. Ahmed, A. Ozcelik, N. Bojanala, N. Nama, A. Upadhyay, Y. Chen, W. Hanna-Rose, and T. J. Huang, Nat. Commun. 7, 11085 (2016).

[25] G. Memoli, M. Caleap, M. Asakawa, D. R. Sahoo, B. W. Drinkwater, and S. Subramanian, Nat. Commun. 8, 14608 (2017).

[26] K. Melde, A. G. Mark, T. Qiu, and P. Fischer, Nature (London) 537, 518 (2016).

[27] A. Riaud, M. Baudoin, O. B. Matar, L. Becerra, and J.-L. Thomas, Phys. Rev. Applied 7, 024007 (2017).

[28] J. Reboud, R. Wilson, Y. Zhang, M. H. Ismail, Y. Bourquin, and J. M. Cooper, Lab Chip 12, 1268 (2012).

[29] J. Reboud, Y. Bourquin, R. Wilson, G. S. Pall, M. Jiwaji, A. R. Pitt, A. Graham, A. P. Waters, and J. M. Cooper, Proc. Natl. Acad. Sci. U.S.A. 109, 15162 (2012).

[30] Y. Bourquin, R. Wilson, Y. Zhang, J. Reboud, and J. M. Cooper, Adv. Mater. 23, 1458 (2011).

[31] J. Shi, D. Ahmed, X. Mao, S.-C. S. Lin, A. Lawit, and T. J. Huang, Lab Chip 9, 2890 (2009).

[32] M. C. Jo and R. Guldiken, Sens. Actuators A 207, 39 (2014).

[33] C. Witte, J. Reboud, R. Wilson, J. Cooper, and S. Neale, Lab Chip 14, 4277 (2014).

[34] Z. Ma, D. J. Collins, and Y. Ai, Anal. Chem. 88, 5316 (2016).

[35] Z. Ma, D. J. Collins, J. Guo, and Y. Ai, Anal. Chem. 88, 11844 (2016).
[36] L. Schmid, D. A. Weitz, and T. Franke, Lab Chip 14, 3710 (2014).

[37] V. Skowronek, R. W. Rambach, L. Schmid, K. Haase, and T. Franke, Anal. Chem. 85, 9955 (2013).

[38] Y. Bian, F. Guo, S. Yang, Z. Mao, H. Bachman, S.-Y. Tang, L. Ren, B. Zhang, J. Gong, X. Guo, and T. J. Huang, Microfluid. Nanofluid. 21, 132 (2017).

[39] D. Psaltis, S. R. Quake, and C. Yang, Nature (London) 442, 381 (2006).

[40] I. Leibacher, P. Reichert, and J. Dual, Lab Chip 15, 2896 (2015).

[41] I. Leibacher, S. Schatzer, and J. Dual, Lab Chip 14, 463 (2014).

[42] P. Augustsson, J. T. Karlsen, H.-W. Su, H. Bruus, and J. Voldman, Nat. Commun. 7, 11556 (2016).

[43] C. Devendran, D. J. Collins, Y. Ai, and A. Neild, Phys. Rev. Lett. 118, 154501 (2017).

[44] G. Destgeer, K. H. Lee, J. H. Jung, A. Alazzam, and H. J. Sung, Lab Chip 13, 4210 (2013).

[45] D. J. Collins, C. Devendran, Z. Ma, J. W. Ng, A. Neild, and Y. Ai, Sci. Adv. 2, e1600089 (2016).

[46] See Supplemental Material at http://link.aps.org/ supplemental/10.1103/PhysRevLett.120.074502 for Huygens-Fresnel concepts, experimental details and additional results.

[47] S. M. Kramer, S. L. McBride, H. D. Mair, and D. Hutchins, IEEE Trans. Ultrason. Ferroelectr. Freq. Control 35, 253 (1988).

[48] F. Kiebert, S. Wege, J. Massing, J. König, R. Weser, H. Schmidt, and C. Cierpka, Lab Chip 17, 2104 (2017).

[49] J. Weight and A. Hayman, J. Acoust. Soc. Am. 63, 396 (1978).

[50] A. Hayman and J. Weight, J. Acoust. Soc. Am. 66, 945 (1979).

[51] D. J. Collins, T. Alan, and A. Neild, Appl. Phys. Lett. 105, 033509 (2014).

[52] J. Park, J. H. Jung, G. Destgeer, H. Ahmed, K. Park, and H. J. Sung, Lab Chip 17, 1031 (2017).

[53] J. H. Jung, G. Destgeer, J. Park, H. Ahmed, K. Park, and H. J. Sung, Anal. Chem. 89, 2211 (2017).

[54] A. Winkler, R. Brünig, C. Faust, R. Weser, and H. Schmidt, Sens. Actuators A 247, 259 (2016).

[55] G. Destgeer, B. H. Ha, J. Park, J. H. Jung, A. Alazzam, and H. J. Sung, Phys. Procedia 70, 34 (2015).

[56] C. Devendran, T. Albrecht, J. Brenker, T. Alan, and A. Neild, Lab Chip 16, 3756 (2016).

[57] A. N. Nordin and M. E. Zaghloul, IEEE Trans. Microwave Theory Tech. 55, 992 (2007).

[58] M. Antfolk, P. B. Muller, P. Augustsson, H. Bruus, and T. Laurell, Lab Chip 14, 2791 (2014).

[59] O. Jakobsson, C. Grenvall, M. Nordin, M. Evander, and T. Laurell, Lab Chip 14, 1943 (2014).

[60] T. Laurell, F. Petersson, and A. Nilsson, Chem. Soc. Rev. 36, 492 (2007).

[61] J. Shi, H. Huang, Z. Stratton, Y. Huang, and T. J. Huang, Lab Chip 9, 3354 (2009).

[62] A. Lenshof, M. Evander, T. Laurell, and J. Nilsson, Lab Chip 12, 684 (2012).

[63] R. Zhou, F. Bai, and C. Wang, Lab Chip 17, 401 (2017).

[64] D. Di Carlo, Lab Chip 9, 3038 (2009).

[65] S. Yang, J. Y. Kim, S. J. Lee, S. S. Lee, and J. M. Kim, Lab Chip 11, 266 (2011). 